\title{
Claves de identificación de microalgas frecuentes en monumentos
}

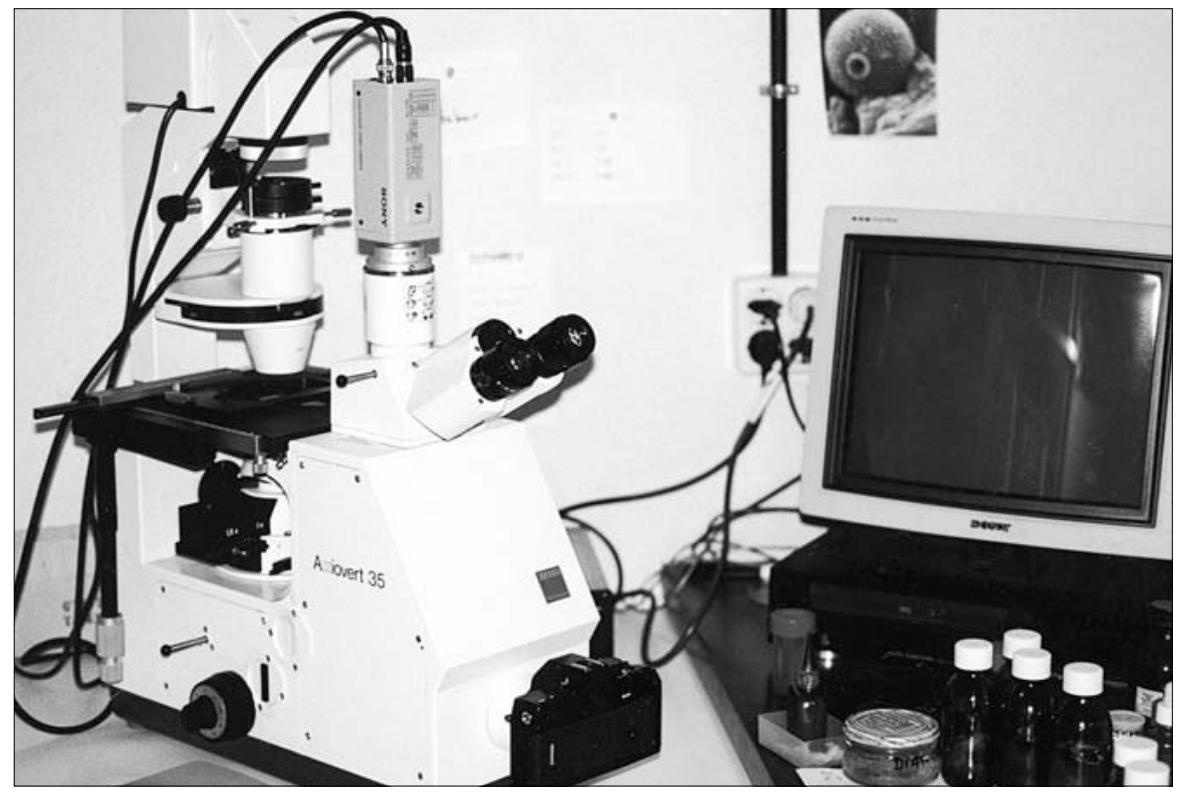

Fernando C. Bolívar Galiano Departamento de Pintura Facultad de Bellas Artes Universidad de Granada

Pedro M. Sánchez Castillo Departamento de Biología Vegetal Facultad de Ciencias Universidad de Granada
Como complemento al artículo aparecido en el número 24 de esta revista (Bolívar \& Sánchez-Castillo 1998: Biodeterioro del patrimonio artístico por cianobacterias, algas verdes y diatomeas), se quiere poner a disposición de todo aquel interesado en determinar la naturaleza algal de las pátinas o formaciones biológicas presentes sobre monumentos, estas claves elaboradas para poder diferenciar los tres grupos principales de microalgas (cianobacterias, algas verdes y diatomeas) e incluso, poder identificar los géneros más implicados en los procesos de biodeterioro, con la ayuda de medios ópticos. Esta clave dicotómica está especialmente indicada para fuentes monumentales, pudiendo ser aplicable en muchos casos también en pinturas murales, cuevas y fachadas.

Otro motivo por el que presentar estas claves, reside en el hecho de no existir hasta la fecha ninguna para la determinación de las microalgas presentes en los monumentos. Del mismo modo, son muy poco 
$\longleftarrow$
$\square$ abundantes las claves en español de algas de ambientes naturales (ríos y lagunas), estando la mayor parte de ellas en idiomas de difícil manejo, como el alemán y el polaco, siendo más escasas y menos precisas algunas en ingles y francés. Esto se debe a la escasez de especialistas dedicados a la taxonomía y estudio de las algas en España y los países hispanoparlantes, lo cual va ligado también a una ausencia de traducciones al castellano.

Por ello, a continuación se presentan las siguientes claves de determinación basadas en caracteres tanto macroscópicos como microscópicos, que esperamos pueda ser de utilidad para todos los profesionales de la conservación. Cada género esta ilustrado en las láminas de iconos agrupados por biotipos y realizadas a partir nuestras observaciones al microscopio óptico de muestras procedentes de diversos monumentos. Las fotografías citadas se refieren a las aparecidas en el artículo antes citado. Para los caracteres macroscópicos se aconseja consultar el artículo: Sánchez-Castillo \& Bolívar, 1997: Caracterización de comunidades algales epilíticas en fuentes monumentales y su aplicación a la diagnosis del biodeterioro. Limnética, 13 (I).

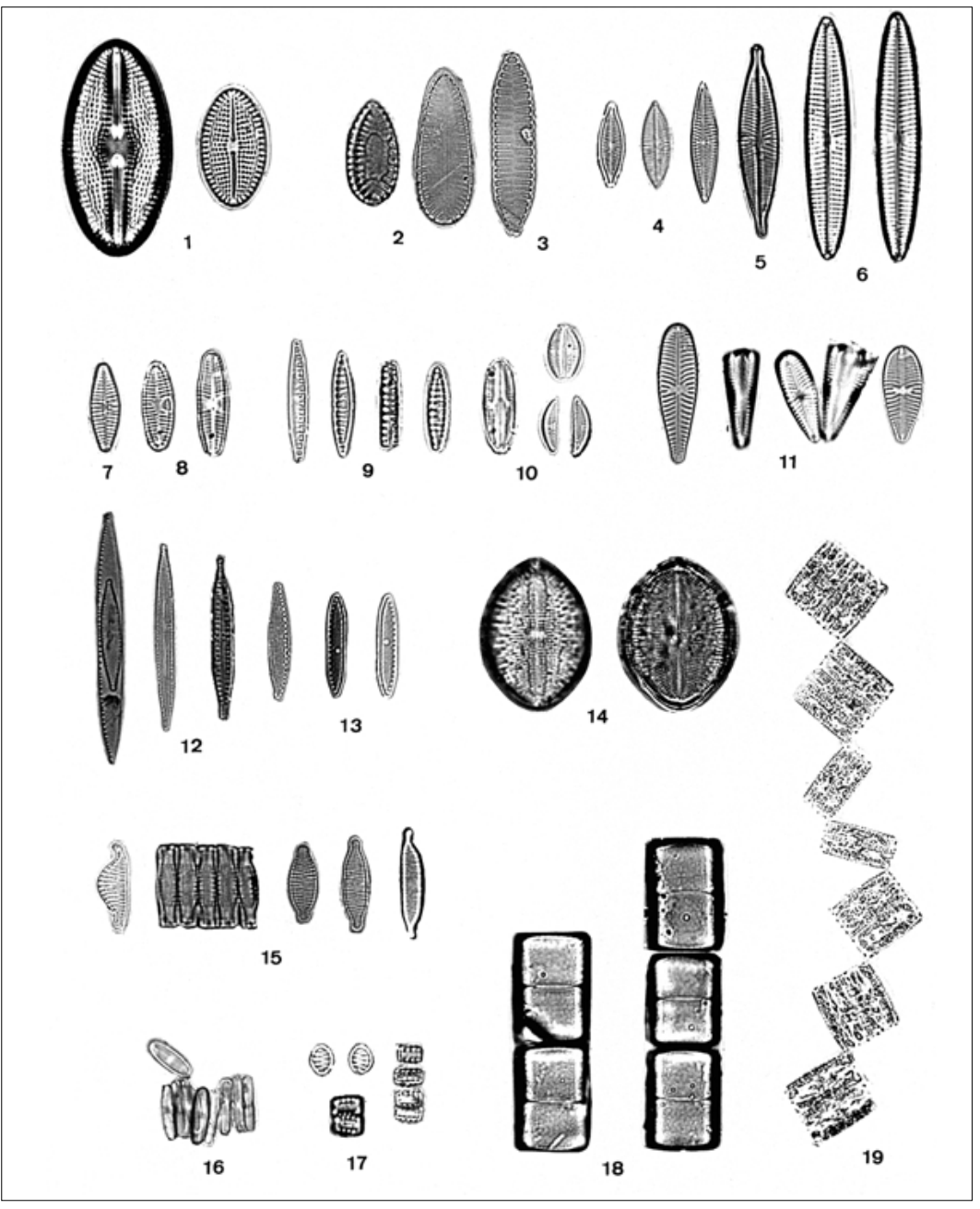

Lám. 0: DIATOMEAS. I. Diploneis ovalis; 2. Surirella brebissonii; 3. Surirella angusta; 4. Navicula menisculus; 5. Navicula rhyncocephala; 6. Navicula gracilis; 7. Achnanthes sp.; 8. Achnanthes lanceolata; 9. Denticula tenuis; 10. Amphora perpusilla; 11. Gomphonema olivaceum; 12. Nitzschia linearis; 13. Nitzschia sp. 4; 14. Cocconeis pediculus; 15. Fragilaria vaucheriae, 16. Navicula seminulum var. fragilarioides; 17. Fragilaria pinnata; 18. Melosira varians; 19. Diatoma vulgare.

\section{DIATOMEAS}

Algas de color marrón rojizo o dorado, células encerradas en una cubierta de naturaleza silícea de alto índice de refracción denominada frústulo: DIATOMEAS. Todas las consideradas a continuación son de vida aislada, entre las de estructura filamentosa solo cabe mencionar a Melosira (Lám.0. I 8; que forma ovas en el interior de las fuentes umbrías), Diatoma (Lám.0.19) y Fragilaria (Lám.0.15, 17) éstas dos últimas formando filamentos que se deshacen fácilmente.

I. Valvas esféricas, de simetría radiada, presentando estrías en su zona marginal Cyclotella

2. Valvas elípticas, lanceoladas u oblongas, presentando rafe (fisura longitudinal) y estrías. Valvas bisimétricas o monosimétricas.

2.I. Márgenes de las valvas diferentes, uno convexo y el otro recto o poco curvado, rafe paralelo al margen recto Cymbella

\subsection{Ambas valvas convexas}

\subsection{Visión comisural no recta}

a) Visión comisural acodada Achnanthes (Lám.0.7,8)

b) Visión comisural trapezoidal Gomphonema (Lám.0.1 I)

\subsubsection{Visión comisural recta}

a) Visión valvar elíptica u oblonga Amphora (Lám.0. 10; Foto 8, bandas intercalares y estrías imperceptibles), Cocconeis (Lám.0.I4; bandas longitudinales que recorren toda la valva), Diploneis (Lám.0.I; estriación formada por areolas), SurireIla (Lám.0.2,3; gruesas estrías y claro canal central), Cymatopleura (estrangulamiento central y margen ondulado).

b) Visión valvar lanceolada Navicula (Lám.0.4-6, I 6; con estrías repartidas a ambos lados del rafe), Nitzschia (Lám.0.12, 13; Fotos 8 y 9, valvas de aspecto hialino, con puntuaciones laterales), Synedra (valvas en forma de aguja con estrías muy finas y paralelas), Denticula (Lám.0.9).

\section{II}

\section{ALGAS VERDES}

Células de color verde intenso, rodeadas de pared celular o no: ALGAS VERDES (y Euglena)

I. Organismos de vida aislada, no unidos en agrupaciones celulares.

\section{I.I. Células móviles mediante flagelos.}

I.I.I. 2 flagelos y superficie celular rígida Chlamydomonas (Lám. I.A) 
I.I.2. I flagelo y superficie deformable Euglena

I.2. Células inmóviles o con movimiento muy lento no producido por flagelos

I.2.I. Células esféricas, con una marcada diferencia de tamaño Chlorococcum (Lám. I.B)

\section{I.2.2. Células no esféricas}

a) Células elípticas Oocystis (superficie lisa, a veces agrupada), Scotiellopsis (Lám. I.C; Foto 7, costillas longitudinales de polo a polo).

b) Células de otra forma, presentando una sutura o estrangulamiento central que las divide en dos fragmentos idénticos Cosmarium (Lám. I.E; células estranguladas) Closterium (Lám. I.D; células falcadas con sutura central).

2. Organismos constituyendo agrupaciones celulares no filamentosas.

2.I. Pequeños grupos de células de 4-8(16) de morfología definida. Células ovales o elipsoidales formando agrupaciones tabulares Scenedesmus (Lám.3.B,C), generalmente con espinas, papilas,verrugas u otro tipo de ornamentación), Poloidion (Lám.3.F; células elíptico-esferoidales con un extremo de la pared engrosado), Tetracystis (Lám.3.D,E; células esféricas de disposición tetraédrica)

2.2. Grandes grupos de células intensamente compactadas Chlorosarcinopsis (Lám.3.G; generalmente angulosas y con un pirenoide muy patente), Fernandinella (Lám.3.H; con diferenciación polar)

3. Algas verdes filamentosas o pseudoparenquimáticas.

3.I. Pequeños filamentos microscópicos unidos (pseudoparénquimas) que tapizan la superficie de las piedras.

3.I.I. Filamentos escasos y poco desarrollados, normalmente en paquetes cúbicos o irregulares Apatococcus (Lám.5.A-C)

3.1.2. Filamentos con ramas anastomosadas, quedando libres solamente los ápices de las mismas Endoderma (Lám.5.D; parásita de otras algas), Pleurastrum (Lám.5.G,H)

3.I.3. Filamentos erguidos y rastreros Leptosira (Lám.5.F; morfología netamente filamentosa, sin pirenoide), Gongrosira (Lám.5.E; parte basal de tipo parenquimático, con pirenoide), Pseudopleurococcus (Lám.5.l; ramas laterales muy poco desarrolladas).

3.2. Filamentos de mayor tamaño (micro-macroscópicos) que viven erguidos sobre la superficie de las piedras o de otras algas y pueden formar ovas.

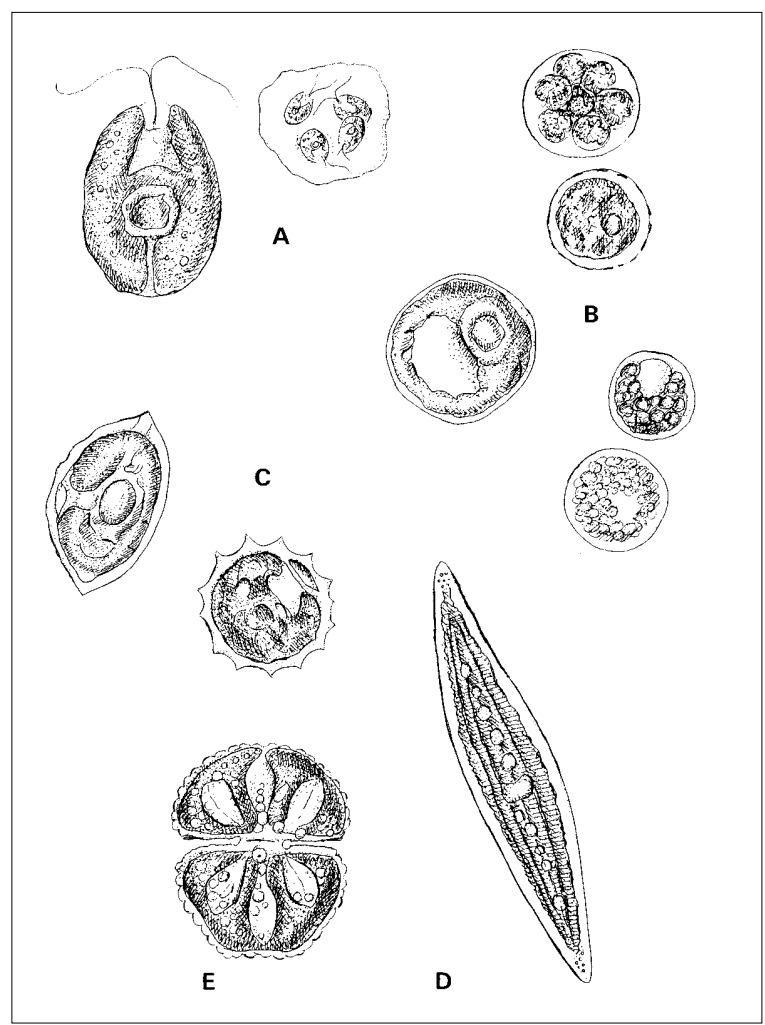

Lám. I: Dibujos a partir de observaciones al Microscopio Óptico de las microalgas verdes del biotipo l: Unicelular. A) Chlamydomonas snowiae; B) Chlorococcum spp.; C) Scotiellopsis terrestris; D) Closterium acerosum; E) Cosmarium botrytis.

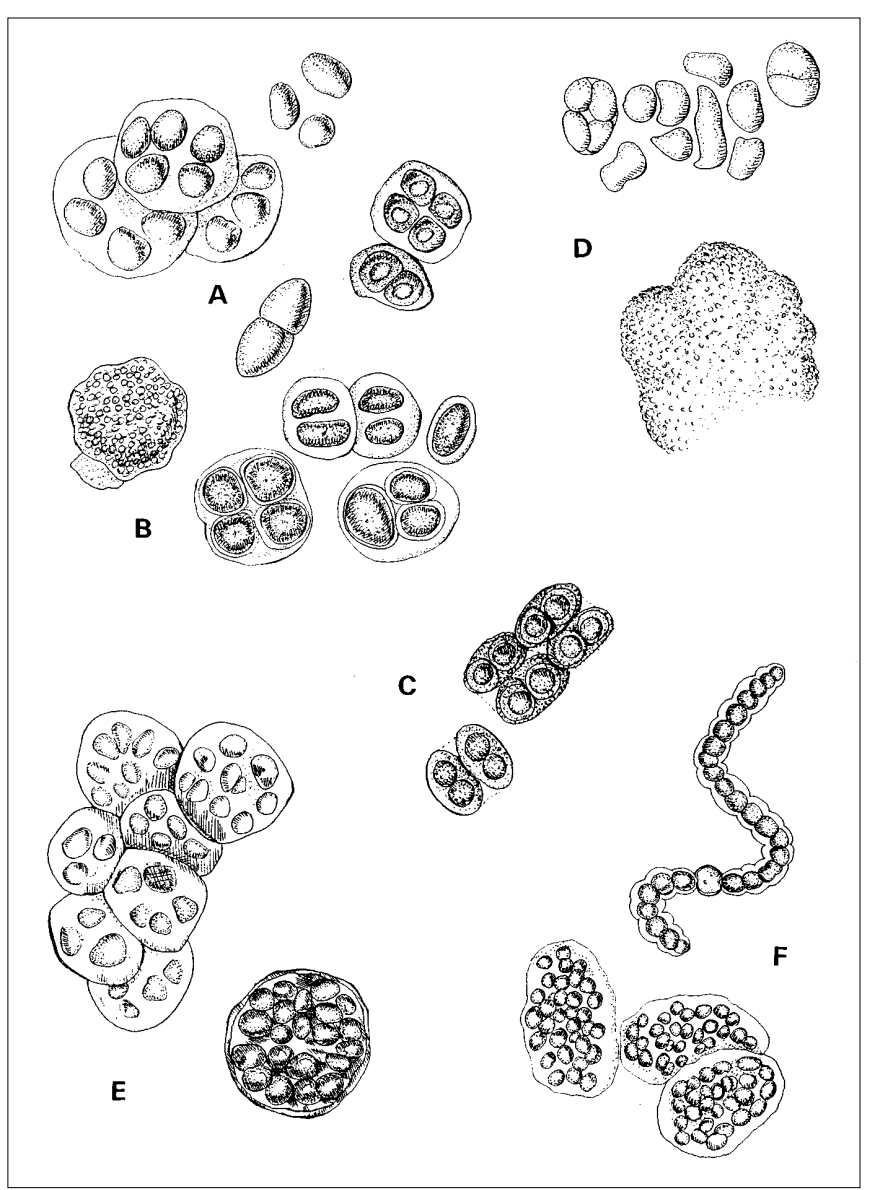

Lám. 2: Cianobacterias del biotipo II: Agregado gelatinoso. A y B) Aphanothece saxicola; C) Gloeocapsa compacta; D) Chlorogloea microcystoides. E) Chroococcidiopsis sp. F) Nostoc sp. 


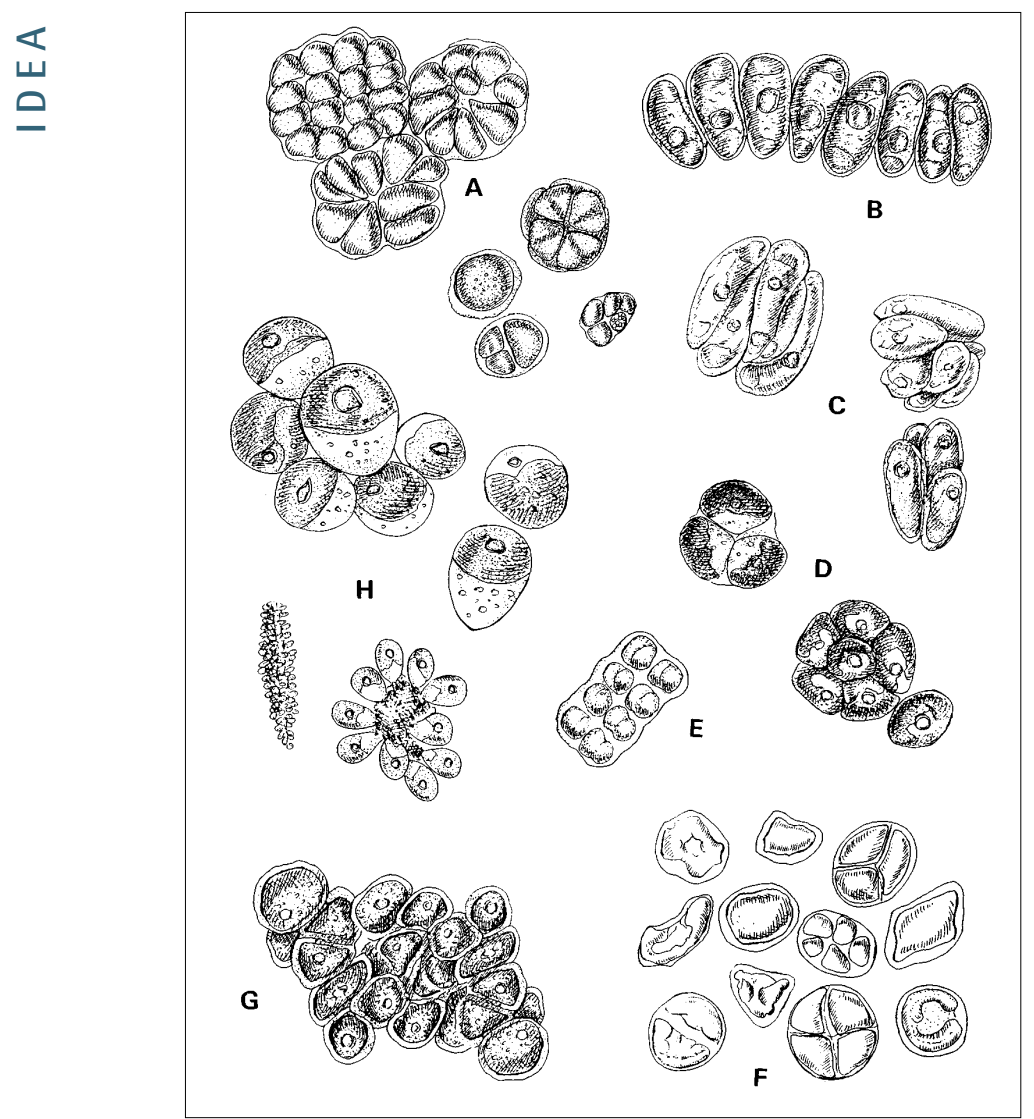

Lám. 3: Biotipo III: Agregado no gelatinoso. A) Myxosarcina chroococcoides; B) Scenedesmus ecornis. C) Scenedesmus smithii. D) Tetracystis sp. E) Tetracystis sarcinalis. F) Poloidion didymos. G) Chlorosarcinopsis minor. H) Fernandinella alpina.

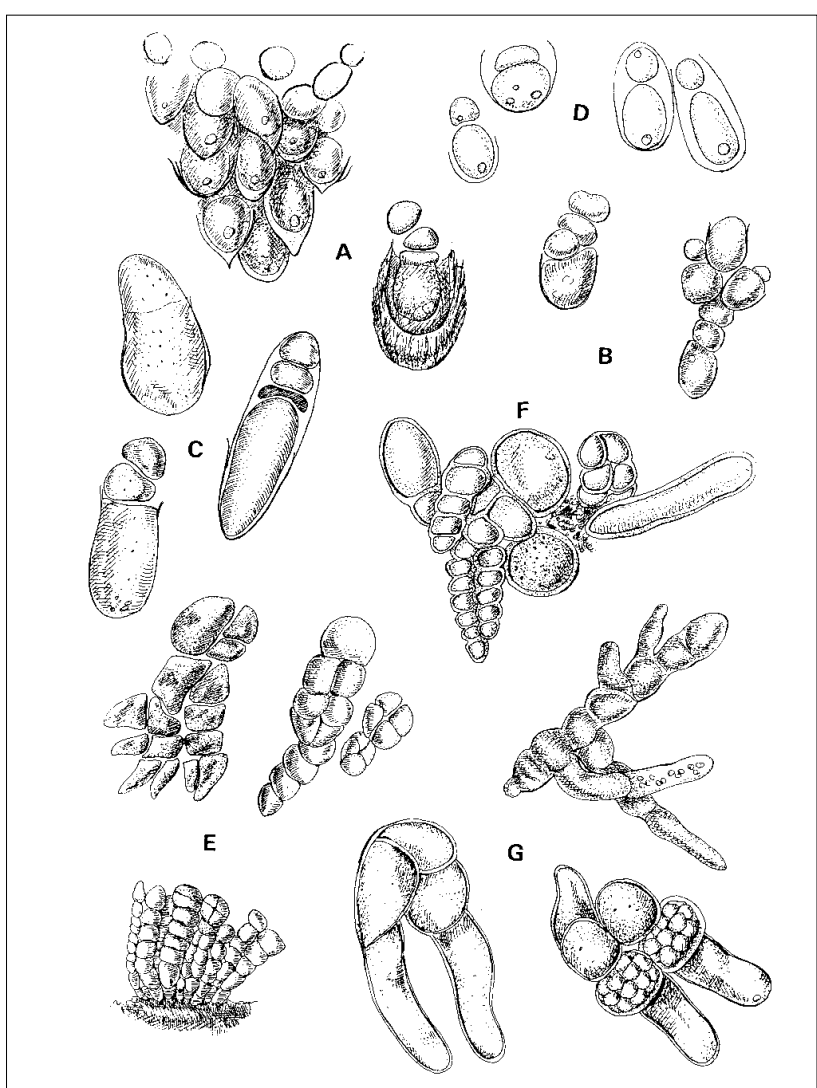

Lám. 4: Cianobacterias del biotipo IV: Estructura pseudoparenquimática. A) Chamaesiphon polonicus; B) Chamaesiphon polymorphus. C) Chamaesiphon incrustans. D) Ch. oncobyrsoides. E) Pleurocapsa minor. F) Pleurocapsa fluviatilis. G) Hyella fontana.
3.2.I Filamentos no ramificados Cylindrocapsa (Lám.6.C; gruesas paredes celulares y capacidad desarticulante), Microspora (presencia de artejos en forma de $\mathrm{H}$ entre células contiguas), Oedogonium (plastos reticulados y anillos de crecimiento), Spirogyra (plastos en forma de espiral), Ulothrix (gran cantidad de células con plastos parietales)

3.2.2. Filamentos ramificados Cladophora (Lám.6.B; pared celular estratificada, formando grandes masas de ovas), Stigeoclonium (Filamentos que acaban muy estrechados, en forma de pelo)

\section{III}

\section{ALGAS VERDE AZULADAS}

Algas de un característico color verde azulado, presentando vainas o aglutinándose por sus paredes celulares. ALGAS VERDE AZULADAS (cianobacterias).

I. Agrupaciones mucilaginosas formando grupos aislados o mezcladas con otras algas.

I.I. Agrupaciones no formadas por filamentos típicos Aphanothece (Lám.2.A,B; células elipsoidales con vainas homogéneas), Gloeocapsa (Lám.2.C; células redondeadas con vainas concéntricas), Chlorogloea (Lám.2.D; células de contorno irregular con cierta tendencia a agruparse en haces longitudinales), Chroococcidiopsis. (Lám.2.E; células rodeadas por vaina coloreada)

I.2. Agrupaciones constituidas por filamentos Nostoc (Lám.2.F; filamentos arrosariados envueltos por vainas mucilaginosas)

2. Agrupaciones de carácter compactante o tapizantes.

2.I. Células unidas formando paquetes microscópicos de contorno anguloso Myxosarcina (Lám.3.A)

2.2. Células unidas formando cortos filamentos o pequeñas láminas (pseudoparénquimas)

2.2.I. Células con vainas coloreadas y productoras de exosporas, forman manchas marrones muy oscuras Chamaesiphon (Lám.4.A-D)

2.2.2. Células poligonales formando filamentos sin ramificar o con ramas poco desarrolladas con zona rizoidal diferenciada Pleurocapsa (Lám.4.E,F)

2.2.3. Células formando filamentos claramente ramificados Hyella (Lám.4.G)

2.3. Células unidas constituyendo filamentos sin vaina.

2.3.I. Filamentos móviles que pueden dar lugar a ovas Oscillatoria (Lám.6.A)

2.4. Células unidas constituyendo filamentos englobados en vainas. 
2.4.I. Heterocistos en la base de filamentos polares

a) Filamentos sin formar falsas ramificaciones Calothrix (Lám.7.A,B)

b) Filamentos formando falsas ramificaciones Tolypothrix (Lám.7.C)

2.4.2. Sin heterocistos

a) Un sólo tricoma por vaina

a. I) Vaina mucosa no excesivamente gruesa, coalescente a veces no diferenciable constituyendo una matriz gelatinosa. Célula apical generalmente diferenciada, muy variable. tapetes de morfología variable, formados por filamentos irregularmente entrelazados o formando haces paralelos al sustrato Phormidium (Lám.8.A-G)

a.2) Vaina consistente. Células apicales, por lo general no diferenciables. Tapetes de morfología variable.

- Tapetes formados por una base de filamentos postrados que en la periferia constituyen haces erectos de forma cónica, con ápices no capitados y sin cofia (tricoma menos de 9 micras) Symploca (Lám.8.J,K)

- Tapetes constituidos únicamente por filamentos postrados

- Filamentos fuertemente entrelados, a veces con falsas ramificaciones, vainas amplias a veces con más de un tricoma Schizothrix-Hypheothrix- (Lám.8.H,I)

- Filamentos escasamente entrelazados constituyendo tapetes muy poco consistentes, generalmente entre concreciones minerales. Célula apical redondeada Lyngbya (Lám.8.D-F)

b) Varios tricomas por vaina

b. I.) Numerosos tricomas por vaina; la vaina es mucosa y a menudo se deshace en el ápice Microcoleus (Lám.7.H,I)

b.2.) Pocos (o sólo uno) tricomas por vaina.

- Vaina mucosa, deshecha en los individuos más viejos, célula apical, normalmente, engrosada y con cofia Hydrocoleus

- Vaina firme, nunca deshecha. Célula apical sin cofia engrosada Schizothrix (Lám.8.H,I)

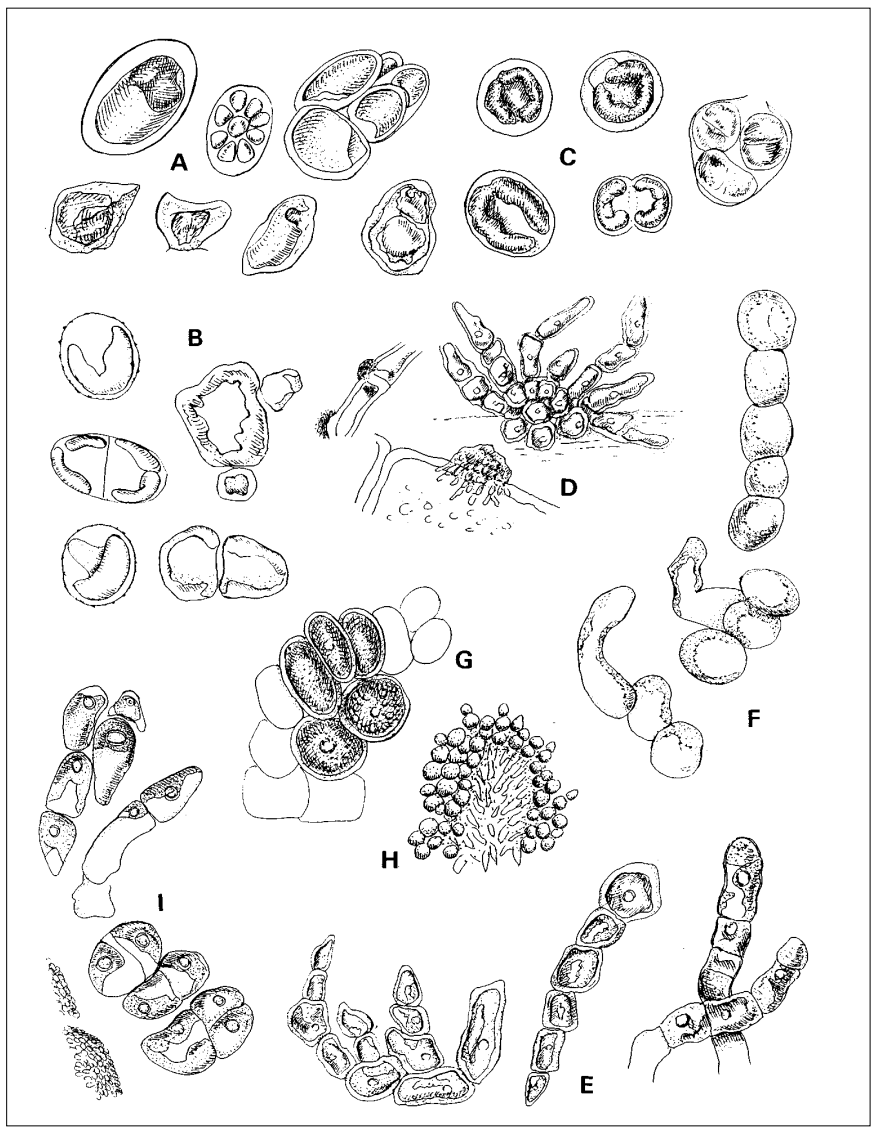

Lám. 5: Algas verdes del biotipo IV: Estructura pseudoparenquimática. A) Apatococcus lobatus. B) Apatococcus vulgaris. C) Apatococcus sp. D) Endoderma cladophorae. E) Gongrosira spp. F) Leptosira trombii. G y H) Pleurastrum spp. I) Pseudopleurococcus sp.

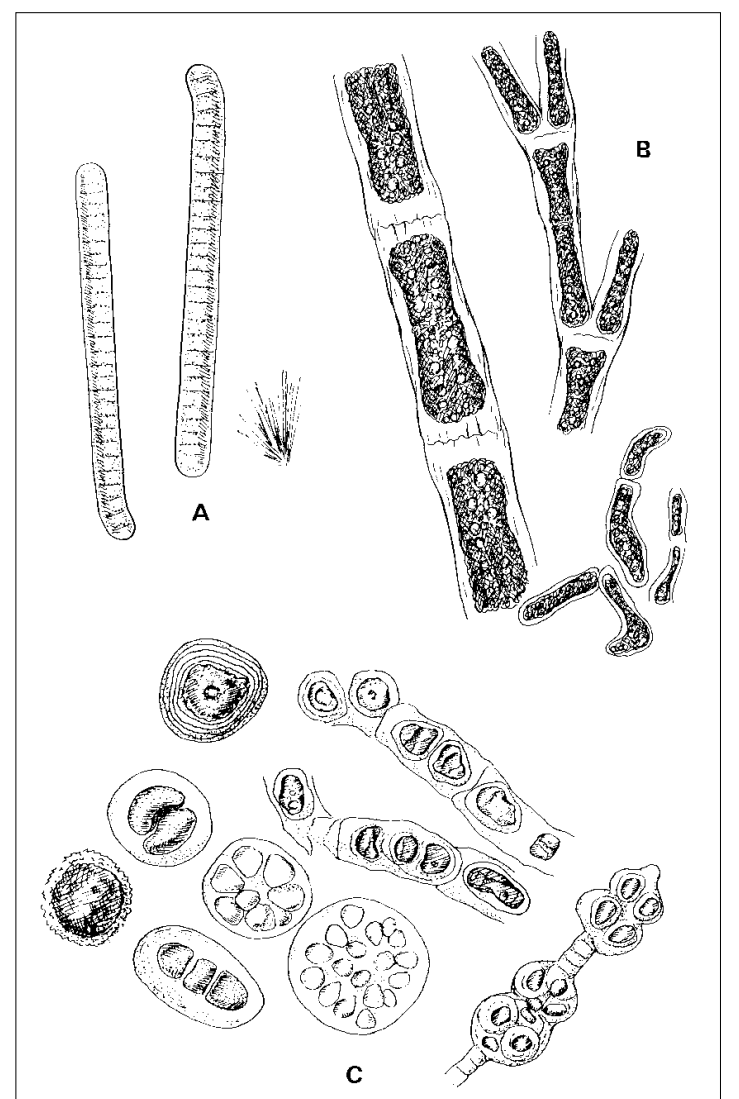

Lám. 6: Biotipo V: Filamentos (sin vaina). A) Oscillatoria sp. B) Cladophora glomerata. C) Cylindrocapsa sp. 


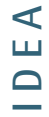

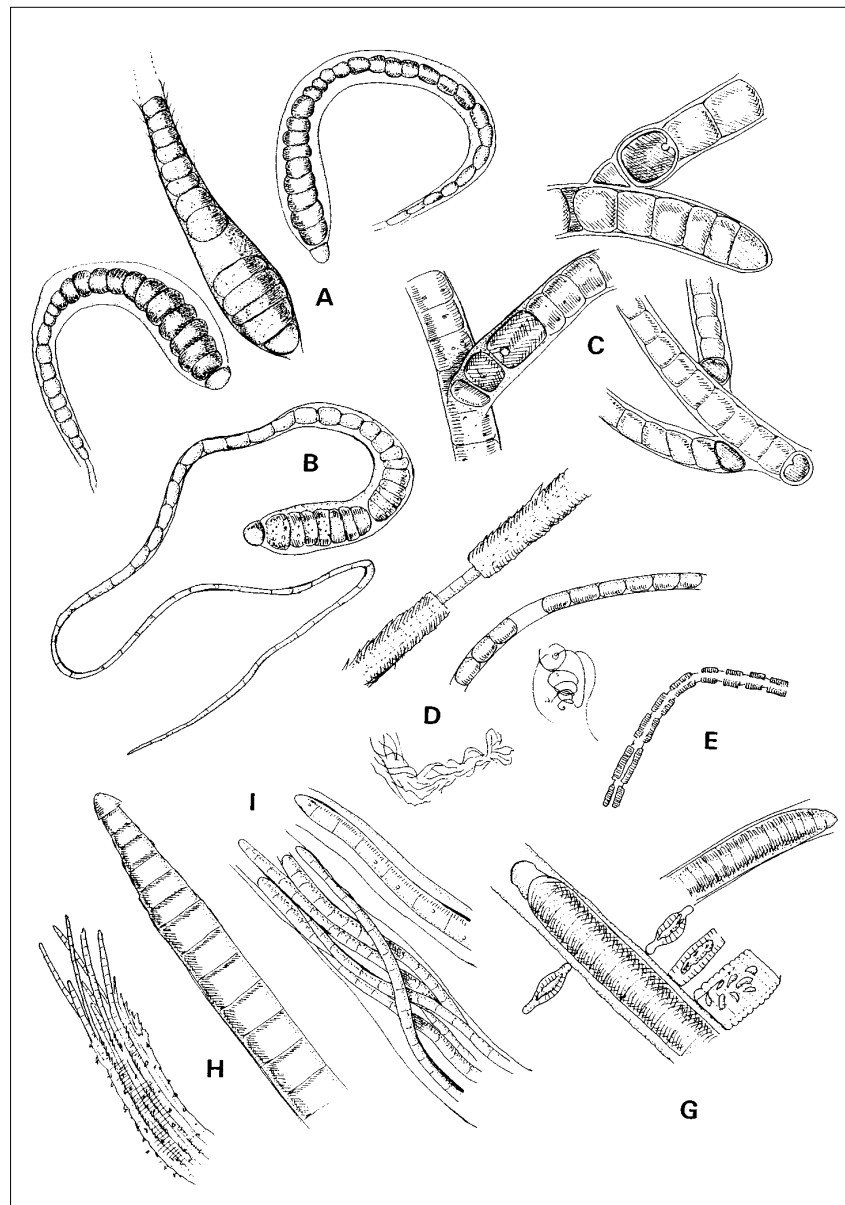

Lám. 7: Cianobacterias del biotipo $\mathrm{V}$ : Filamentos (con vaina). A) Calothrix elenkinii. B) Calothrix sp. C) Tolypothrix sp. D) Lyngbya amplivaginata. E) Lyngbya lagerheimii. F) Lyngbya martensiana. G) Lyngbya nigra. H) Microcoleus vaginatus. I) Microcoleus sp.

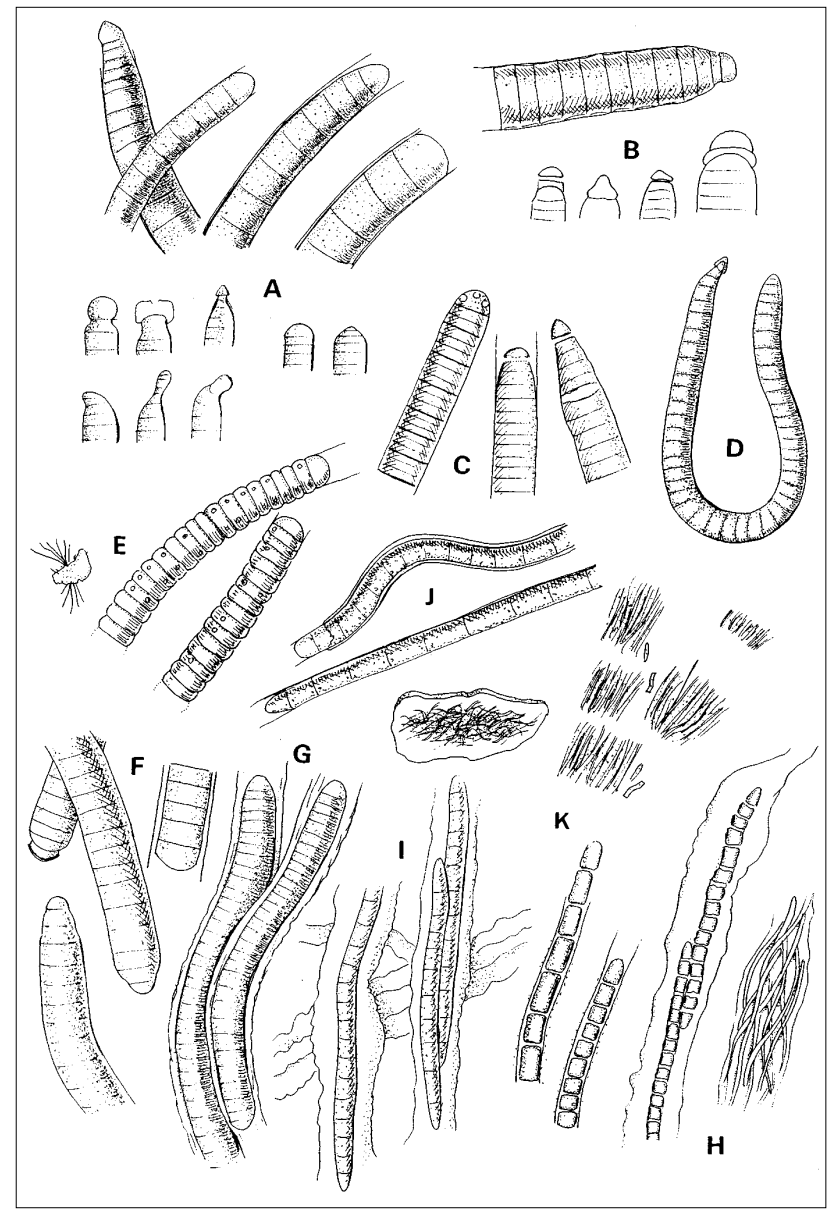

Lám. 8: Otras cianobacterias del biotipo V: Filamentos (con

vaina). A) Phormidium favosum

B) Phormidium subfuscum C)

Phormidium subfuscum var. ina-

equale. D) Phormidium uncina-

tum. E) Phormidium ambiguum

F) Phormidium calidum G)

Phormidium retzii $H$ )

Schizothrix gomonti. I)

Schizothrix sp. J) Symploca

muralis. K) Symploca elegans. 